\title{
Adaptation in polymerase basic protein 1 family from influenza $A$ virus to climate change
}

\author{
Shaomin Yan, Guang Wu
}

State Key Laboratory of Non-Food Biomass Enzyme Technology, National Engineering Research Center for Non-Food Biorefinery, Guangxi Key Laboratory of Biorefinery, Guangxi Academy of Sciences, Nanning, China; hongguanglishibahao@yahoo.com

Received 13 September 2012; revised 11 October 2012; revised 23 October 2012

\begin{abstract}
Global climate changes affect the functioning of ecosystems, in particular host-pathogen interactions, with major consequences in health ecology, however, it is less addressed how the change in global temperature affects the protein family of influenza virus. In this study, we studied the adaptation of polymerase basic protein 1 (PB1) family from influenza $A$ virus to temperature change. 3841 PB1 proteins sampled from 19562011 were quantified by the amino-acid pair predictability and then compared their general changes with the temperature changes (HadCRUT3v and CRUTEM4v data sets) of corresponding years on a $5^{\circ}$ by $5^{\circ}$ grid-box basis. Also, point-to-point comparisons were conducted from 1956 to 1998 in all and different species. The results showed that both changes in the temperature and unpredictable portion of PB1 proteins had similar trends from 1956 to 2011, which provides the evidence of virus adaptation at protein level to climate change.
\end{abstract}

Keywords: Adaptation; Evolution; Influenza A Virus; Polymerase Basic Protein 1; Temperature Change

\section{INTRODUCTION}

Although climate change imposes serious problems on humans and other species [1], more and more attention is now being paid to the adaptation of different species to climate change at different levels [2-4]. Actually, this type of adaptation goes through mutations, because mutations are the base for either functional or structural adaptation. Nevertheless, adaptation makes a species more suitable to climate change and makes a species survive climate change.

Although mutations benefit a species to adapt to climate change, these mutations could be harmful to other species. This is plausible because mutations would not limit their effects only to adaptation to climate change. Accordingly, any mutation in virus could lead to unexpected effects on human health, for example, unpredictable mutations in influenza A viruses constantly threaten humans with possible deadly pandemics/epidemics. Indeed, not only the mutations whose aim is to adapt to climate change but also the mutations whose aim is to adapt to changed ecosystems, where host-pathogen interactions may change as well $[5,6]$, should be taken into consideration.

On the other hand, virus adaptation is different from the adaptations of many other species because virus lives in host cells with relatively favorable and stable environments. With respect to influenza A virus, climate changes alter the normal pattern of bird migrations, which interrupts the transmission cycle of avian influenza A virus [7], and also influenza A virus can survive outside its living hosts. Therefore, virus mutations would occur more easily than we perceived.

In the past, we studied the adaptation of influenza A viruses to temperature changes with respect to their six proteins, hemagglutinin, neuraminidase, nucleoprotein, polymerase acidic protein, polymerase basic protein 2 , and matrix protein 2 [8-13]. However, there are ten or eleven different proteins in influenza A viruses according to different strains, so it is necessary to study the adaptations in the remaining proteins from influenza A viruses in order to get a whole picture. Moreover, a considerable amount of new data concerning influenza A virus as well as temperature just become available, so it is necessary to incorporate these new data into this type of analysis.

The polymerase basic protein 1 (PB1) is a protein from influenza A virus as a subunit of RNA-dependent RNA polymerase complex. It associates with the transcription and replication of the influenza A viral genome [14], so it is important for the efficient propagation of the virus in the host and for its adaptation to new hosts [15]. As a part of influenza A virus, PB1 needs to adapt to any change in its host and its adaptation could lay the foundation for the survival of influenza A virus, of which new strain could lead epidemic as well as pandemic. Nowa- 
days it is considered as a determinant of the pathogenicity of the 1918 pandemic virus [16]. Also, influenza PB1 is the major target for both CD4(+) and CD8(+) T-cell responses [17]. In this study, we analyzed the adaptation of PB1 proteins to temperature change.

\section{MATERIALS AND METHODS}

\subsection{Temperature Data}

The global temperature anomalies from 1850 to 2011, whose anomaly was based on the period 1961-1990, were obtained from HadCRUT3v data set that was the variance adjusted version of combined land and marine temperature anomalies on a $5^{\circ}$ by $5^{\circ}$ grid-box basis and from CRUTEM4v data set that was the variance adjusted version of land air temperature anomalies on a $5^{\circ}$ by $5^{\circ}$ grid-box basis [18,19]. The local temperature from 1956 to 1998 based on $0.5^{\circ}$ by $0.5^{\circ}$ latitude and longitude gridbox basis cross globe was obtained from New et al. [20].

\subsection{PB1 Data}

10732 full-length PB1 proteins of influenza A viruses sampled from 1956-2011 were obtained from the Influenza Virus Resources [21]. All groups of identical sequences in the dataset were represented by the oldest sequence in the group so that 3841 PB1 proteins were actually used in this study.

\subsection{Adaptation in PB1 to Temperature Change}

Virus adaptation is a mutation process along the time course, and interestingly temperature change is a process along the time course, too. These two facts dictate that we can plot both temperature change and virus adaptation along the time course, observe their relationship and determine whether virus adaptation occurs to suit temperature change.

At the level of species, adaptation can refer to features in population of species, for example, the number of population changes along the time course. At the level of individual in species, adaptation can refer to features in an individual in species, for example, the individual's bodyweight changes along the time course. At the level of protein, adaptation can refer to features in a protein family, for example, the amino acid composition changes in protein family along the time course. In agreement with our previous studies along this line [8-13], we used the amino-acid pair predictability as a measure to present PB1's adaptation along the time course [22].

For example, a PB1 protein (accession number BAD89303) has 757 amino acids, which are counted as 756 adjacent amino-acid pairs. There are 49 arginines " $R$ " and 47 isoleucines "I" in this protein. If permutation can predict the appearance of amino-acid pair RI, then it would appear 3 times $(49 / 757 \times 47 / 756 \times 756=3.04)$. Actually it does appear 3 times, so its appearance is predictable. By clear contrast, there were 50 glutamic acids "E" and 48 serines " $S$ " in this PB1 protein. According to the permutation, the amino-acid pair ES would appear 3 times $(50 / 757 \times 48 / 756 \times 756=3.17)$, however, it appeared 7 times in realty, which was unpredictable. In this way, we classified all amino-acid pairs in this protein as predictable and unpredictable portions, which are 28.04\% and $71.96 \%$. For another example, another PB1 protein (accession number BAD89323) has only one amino acid at position 80 different from BAD89303 PB1 protein, and its predictable and unpredictable portions are $27.38 \%$ and $72.62 \%$.

In this manner, each PB1 protein has its own predictable/unpredictable portion, and 3841 PB1 proteins have 3841 different predictable/unpredictable portions. As each PB1 protein had its sampling year, so we can plot either predictable portion or unpredictable portion as a number along the time course to compare with the temperature trend over time.

\subsection{Statistics}

Linear regression was used to correlate the relationship between temperate anomaly and time and between unpredictable portion and time. In this way, the regressed lines can be used to observe the trends, which should be considered as the first stage of studies, because more sophisticated methods should be applied after obtaining the confirmative results from the first stage.

\section{RESULTS}

Figure 1 shows the similar trends in temperate change and in PB1 adaptation, which represents as averaged unpredictable portion for each year. For example, there are 3 PB1 proteins in 1956 and 53 PB1s in 2008. Statistically, the data are unbalanced because of difficulties in collection of samples. Consequently this would partially contribute to the difference between three regressions in upper, middle and lower panels.

Still, we can use the point-to-point method to furthermore analyze the trends in Figure 1, that is, we couple each PB1 protein with the temperature recorded at the same place and at same year, where and when that PB1 was sampled. For example, a PB1 protein (accession number AAA43641) was sampled in 1956 at Beijing, whose latitude and longitude are $39.91^{\circ}$ and $116.40^{\circ}$ according to Get Lat Lon [23], where the average yearly temperature was $10.93^{\circ} \mathrm{C}$ in 1956 according to the $0.5^{\circ}$ by $0.5^{\circ}$ latitude and longitude grid-box basis cross globe obtained from New et al. [20].

Figure 2 displays 787 point-to-point relationships be- 


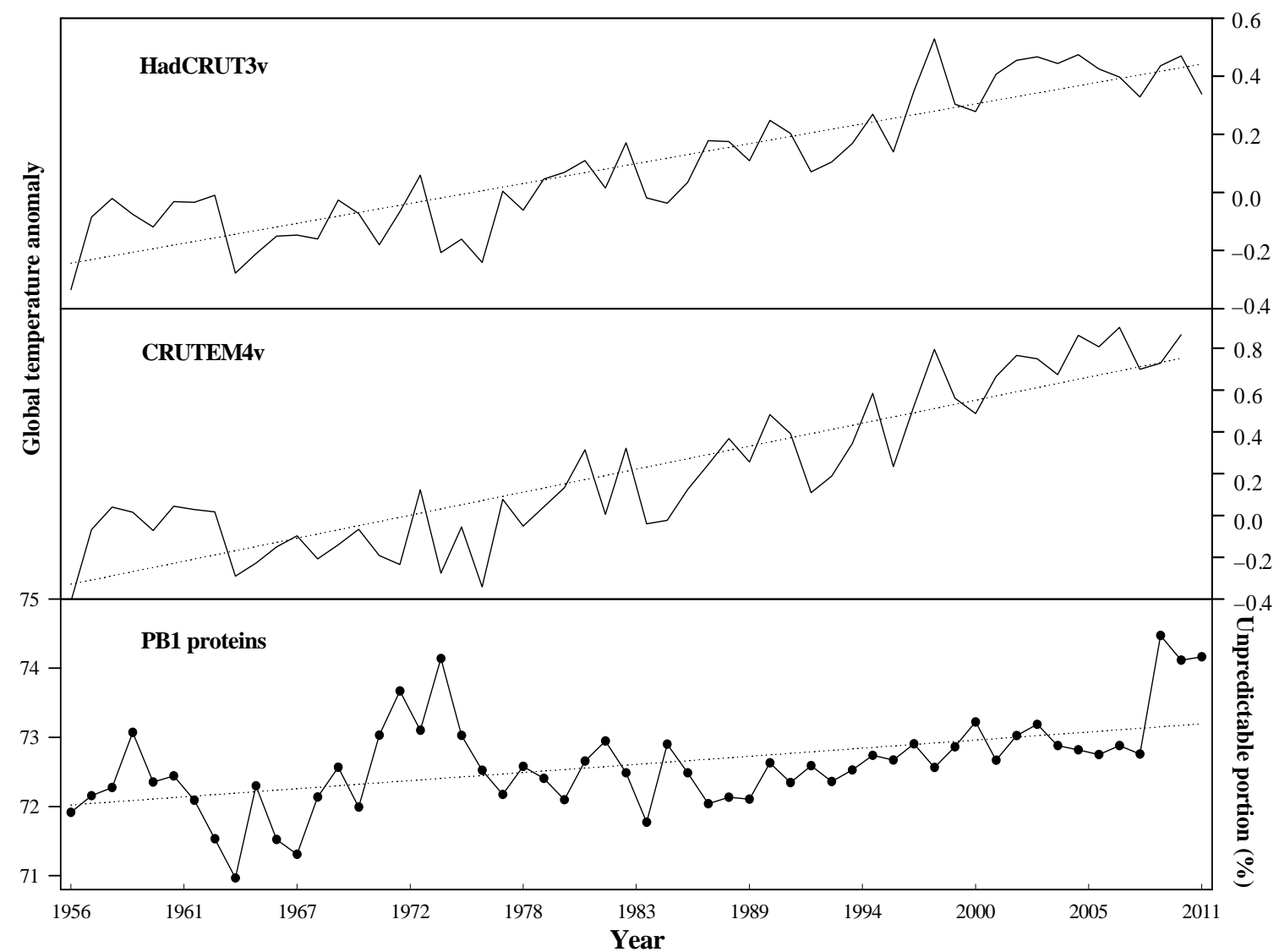

Figure 1. Global temperature anomaly $\left({ }^{\circ} \mathrm{C}\right)$ presented by HadCRUT3v data (upper panel) and by CRUTEM4v data (middle panel), and PB1 adaptation (lower panel). The dotted lines and points were regressed lines and the mean of all PB1 proteins at a given year $(\mathrm{n}=3841$ from 1956 to 2011).

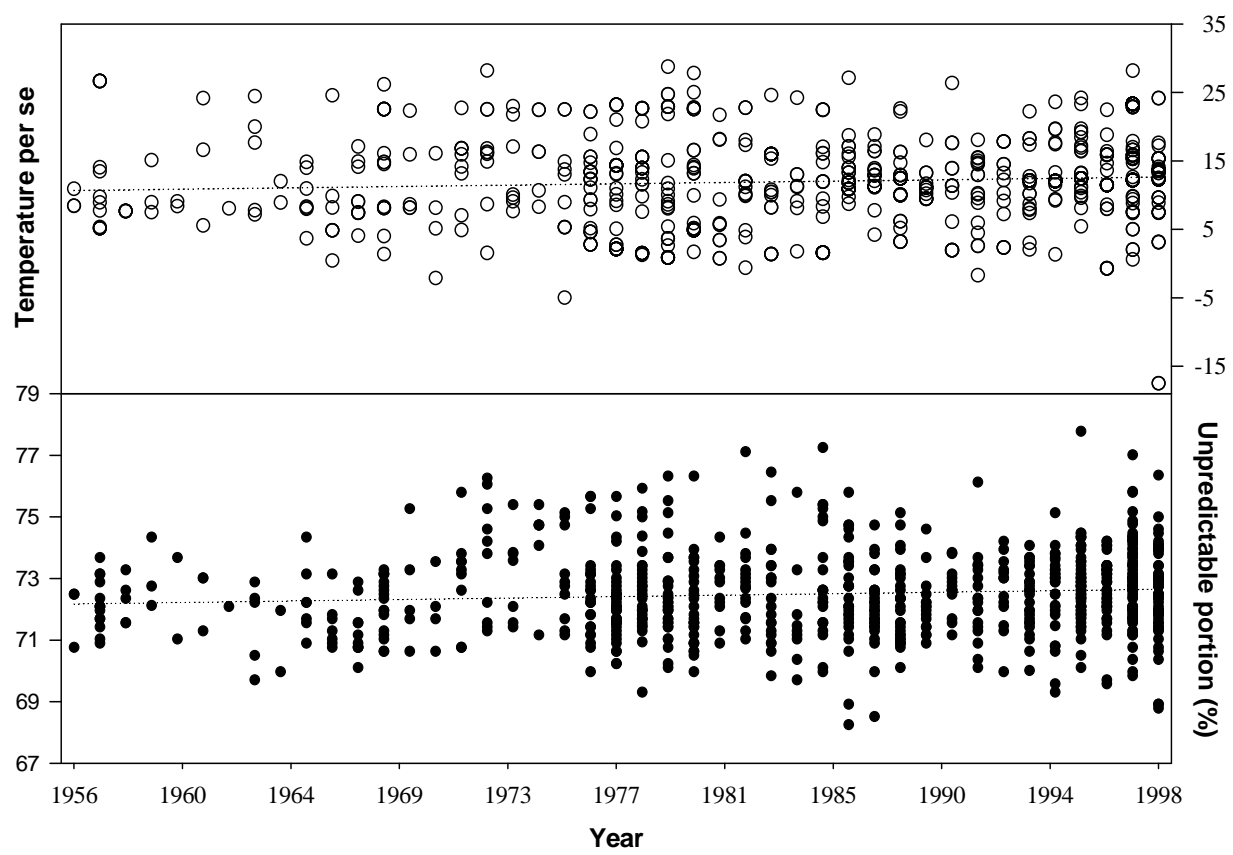

Figure 2. Point-to-point temperature versus PB1 protein $(n=787)$ from 1956 to 1998. Each point presented a local temperature $\left({ }^{\circ} \mathrm{C}\right.$ ) at the given year (upper panel), corresponding to the place where a PB1 protein was sampled (lower panel). The dotted lines were regressed lines. 
tween temperature and unpredictable portion of PB1 proteins from 1956 to 1998, and their regression indicated the similar trends, which shows the trends more clearly than those in Figure 1.

As influenza viruses are hosted in different species, we can advance our knowledge using the point-to-point rela- tionship between temperature and species, where PB1 proteins were sampled. Figure $\mathbf{3}$ shows the PB1 adaptation in three major species with respect to the temperature, where we can see that the trends are similar for PB1 proteins hosted in human and swine, but different in avian.

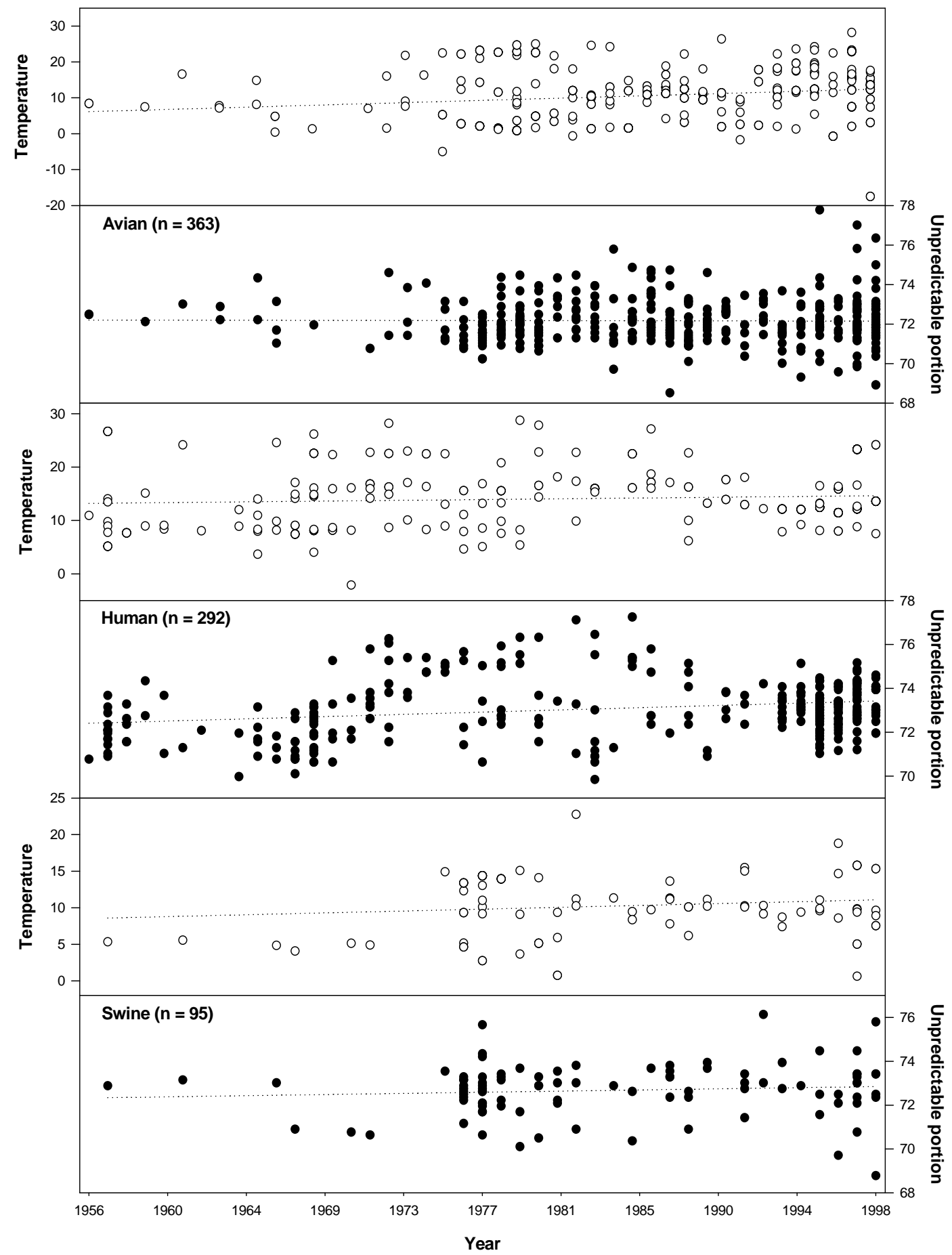

Figure 3. Point-to-point temperature $\left({ }^{\circ} \mathrm{C}\right)$ versus PB1 protein sampled from different species. The dotted lines were regressed lines. 


\section{DISCUSSION}

Both climate change and influenza are very important to human society, and huge efforts were made to determine the influence of climate change on various species. Doubtless, the better understanding of adaptation of influenza A virus would lead us to better understand the structure of virus [24,25], and search for antiviral drugs [26-28]. Arguably this study shows similar trend in both temperature change and virus adaptation, suggesting that temperature change has either direct or indirect effect on adaptation of proteins. In case of proteins from influenza A viruses, they could be exposed outside their host although they replicate inside cells of host, therefore climate change could mostly be indirect and partly direct.

First, the trend in adaptation suggests that PB1's unpredictable portion increases over time, which is parallel to the trend in temperature. In our definition, the unpredictable portion represents the amino-acid pairs whose appearance cannot be predicted by permutation, while a predictable amino-acid pair would have the biggest probability for construction, which is identical with parsimony of nature [22]. Thus, nature should have sufficient reason to spend more time and energy to construct the amino-acid pairs whose appearance is unpredictable. In context of temperature change, the increase in unpredictable portion would suggest that nature does its best to suit climate change by taking more time and energy to construct unpredictable amino-acid pairs.

Second, we can see a somehow lag time between temperature change and PB1's adaptation in reference to several peaks. This lag time is convincing as it exactly suggests the indirect effect of climate change on PB1 proteins. We can understand that hot weather comes at first, and then mutations follow. Still, the fluctuation of unpredictable portion reflects the difference between proteins that is attributed to mutations.

Third, many our previous studies on mutation show that nature has the intention to construct amino-acid pairs whose appearance is predictable because of parsimony. Thus, although nature constructed unpredictable aminoacid pairs to suit to temperature change, nature still wants to minimize the difference between predictable and unpredictable amino-acid pairs. This leads to the fluctuation of average data line in Figure 1.

This study demonstrated the changes in the unpredictable portions of PB1 proteins were different in different species. In human and swine, the trends PB1's adaptation were similar to the trends obtained from temperature change, but not in avian (Figure 3). This difference can be due to the fact that the place where avian was sampled would not be the place where the mutation occurred, because migratory birds are common reservoirs responsible for spreading avian influenza viruses [29,30]. Climate change would almost certainly alter bird migration, influence the avian influenza virus transmission cycle and directly affect virus survival outside the host [7,31]. On the other hand, the human and swine were generally localized, thus the present results indicate the potential impact of climate change on the adaptation of influenza A viruses. On the other hands, the results in this study furthermore support our several previous studies on other proteins of influenza A viruses [8-13]. Nevertheless, more studies are needed in order to better understand these two important issues in front of humans.

As the Darwinian theory suggests that the adaptation of random mutation is due to natural and environmental conditions, climate change may partially chose any mutated influenza A virus that can be easily survive in changed environment. However, it is still not clear to which degree the temperature change contributes to the evolution of influenza A virus because climate change is only one of many factors, which influence the evolutional process of influenza A virus.

\section{ACKNOWLEDGEMENTS}

This study was partly supported by Guangxi Science Foundation (2010GXNSFF013003, 10-046-06 and 11-031-11).

\section{REFERENCES}

[1] Thomas, C.D., Cameron, A., Green, R.E., Bakkenes, M., Beaumont, L.J., Collingham, Y.C., Erasmus, B.F., De Siqueira, M.F., Grainger, A., Hannah, L., Hughes, L., Huntley, B., Van Jaarsveld, A.S., Midgley, G.F., Miles, L., Ortega-Huerta, M.A., Peterson, A.T., Phillips, O.L. and Williams, S.E. (2004) Extinction risk from climate change. Nature, 427, 145-148. doi:10.1038/nature02121

[2] Sears, M.W. and Angilletta Jr., M.J. (2011) Introduction to the symposium: Responses of organisms to climate change: A synthetic approach to the role of thermal adaptation. Integrative and Comparative Biology, 51, 662-665. doi:10.1093/icb/icr113

[3] Shaw, R.G. and Etterson, J.R. (2012) Rapid climate change and the rate of adaptation: Insight from experimental quantitative genetics. New Phytologist, 195, 752-765. doi:10.1111/j.1469-8137.2012.04230.x

[4] Anderson, J.T., Inouye, D.W., McKinney, A.M., Colautti, R.I. and Mitchell-Olds, T. (2012) Phenotypic plasticity and adaptive evolution contribute to advancing flowering phenology in response to climate change. Proceedings of Biological Science, 279, 3843-3852. doi:10.1098/rspb.2012.1051

[5] de La Rocque, S., Rioux, J.A. and Slingenbergh, J. (2008) Climate change: Effects on animal disease systems and implications for surveillance and control. Review on Science and Technology, 27, 339-354.

[6] Gould, E.A. and Higgs, S. (2009) Impact of climate change and other factors on emerging arbovirus diseases. Transaction of Royal Society of Tropic Medicine and Hygiene, 103, 109-121. 
[7] Gilbert, M., Slingenbergh, J. and Xiao, X. (2008) Climate change and avian influenza. Review on Science and Technology, 27, 459-466.

[8] Yan, S. and Wu, G. (2009) What these trends suggest? American Journal of Applied Sciences, 6, 1116-1121. doi:10.3844/ajassp.2009.1116.1121

[9] Yan, S. and Wu, G. (2009) Trends in global warming and evolution of polymerase basic protein 2 family from influenza A virus. Journal of Biomedical Science and Engineering, 2, 458-464.

[10] Yan, S. and Wu, G. (2009) Trends in global warming and evolution of matrix protein 2 family from influenza A virus. Interdisciplinary Science: Computational Life Science, 1, 272-279. doi:10.1007/s12539-009-0053-6

[11] Yan, S.M., Zuo, W.P., Zhu, Q.X., Huang, Y.Y., Pan, L.X. and Wu, G. (2010) Trends in global warming and evolution of neuraminidases from influenza A viruses since 1918. Guangxi Sciences, 17, 80-84.

[12] Yan, S. and Wu, G. (2010) Trends in global warming and evolution of nucleoproteins from influenza A viruses since 1918. Transboundary and Emerging Diseases, 57, 404-413. doi:10.1111/j.1865-1682.2010.01164.X

[13] Yan, S. and Wu, G. (2011) Evidence for possible impact of global warming on evolution of hemagglutinins from influenza A virus. Biomedical and Environmental Sciences, 24, 62-67.

[14] Engelhardt, O.G. and Fodor, E. (2006) Functional association between viral and cellular transcription during influenza virus infection. Review on Medical Virology, 16, 329-345. doi:10.1002/rmv.512

[15] Brower-Sinning, R., Carter, D.M., Crevar, C.J., Ghedin, E., Ross, T.M. and Benos, P.V. (2009) The role of RNA folding free energy in the evolution of the polymerase genes of the influenza A virus. Genome Biology, 10, R18. doi:10.1186/gb-2009-10-2-r18

[16] Watanabe, T., Watanabe, S., Shinya, K., Kim, J.H., Hatta, M. and Kawaoka, Y. (2009) Viral RNA polymerase complex promotes optimal growth of 1918 virus in the lower respiratory tract of ferrets. Proceedings of National Academy of Sciences of United States of America, 106, 588592. doi:10.1073/pnas.0806959106

[17] Assarsson, E., Bui, H.H., Sidney, J., Zhang, Q., Glenn, J., Oseroff, C., Mbawuike, I.N., Alexander, Newman, M.J., Grey, H. and Sette, A. (2008) Immunomic analysis of the repertoire of T-cell specificities for influenza A virus in humans. Journal of Virology, 82, 12241-12251. doi:10.1128/JVI.01563-08

[18] Climatic Research Unit (2012). http://www.cru.uea.ac.uk/cru/data/temperature

[19] Rayner, N.A., Brohan, P., Parker, D.E., Folland, C.K., Kennedy, J.J., Vanicek, M., Ansell, T. and Tett, S.F.B. (2006)
Improved analyses of changes and uncertainties in marine temperature measured in situ since the mid nineteenth century: The HadSST2 dataset. Journal of Climate, 19, 446-469. doi:10.1175/JCLI3637.1

[20] New, M. Hulme M. and Jones, P. (2000) Representing twentieth-century space-time climate variability. Part II: Development of 1901-1996 monthly grids of terrestrial surface climate. Journal of Climate, 13, 2217-2238. doi:10.1175/1520-0442(2000)013<2217:RTCSTC $>2.0 . C$ $\underline{\mathrm{O} ; 2}$

[21] Influenza virus resources (2012). http://www.ncbi.nlm.nih.gov/genomes/FLU/Database/mu ltiple.cgi

[22] Wu, G. and Yan, S. (2008) Lecture notes on computational mutation. Nova Science Publishers, New York.

[23] Get Lat Lon (2012). http://www.getlatlon.com

[24] Pielak, R.M., Oxenoid, K. and Chou, J.J. (2011) Structural investigation of rimantadine inhibition of the AM2-BM2 chimera channel of influenza viruses. Structure, 19, 16551663. doi:10.1016/j.str.2011.09.003

[25] Berardi, M.J., Shih, W.M., Harrison, S.C. and Chou, J.J. (2011) Mitochondrial uncoupling protein 2 structure determined by NMR molecular fragment searching. Nature, 476, 109-113. doi:10.1038/nature 10257

[26] Du, Q.S., Wei, H., Huang, R.B. and Chou, K.C. (2011) Progress in structure-based drug design against influenza A virus. Expert Opinion, 6, 619-631. doi:10.1517/17460441.2011.571671

[27] Li, X.B., Wang, S.Q., Xu, W.R., Wang, R.L. and Chou, K.C. (2011) Novel inhibitor design for hemagglutinin against H1N1 influenza virus by core hopping method. PLoS One, 6, e28111. doi:10.1371/journal.pone.0028111

[28] Wang, S.Q., Cheng, X.C., Dong, W.L., Wang, R.L. and Chou, K.C. (2010). Three new powerful Oseltamivir derivatives for inhibiting the neuraminidase of influenza virus. Biochemistry Biophysics Research Communication, 401, 188-191. doi:10.1016/j.bbrc.2010.09.020

[29] Garamszegi, L.Z. and Møller, A.P. (2007) Prevalence of avian influenza and host ecology. Proceedings of Biological Science, 274, 2003-2012.

[30] Jahangir, A., Watanabe, Y., Chinen, O., Yamazaki, S., Sakai, K., Okamura, M., Nakamura, M. and Takehara, K. (2008) Surveillance of avian influenza viruses in Northern pintails (Anas acuta) in Tohoku District. Japanese Avian Disease, 52, 49-53. doi:10.1637/8035-062507-Reg

[31] Louchart, A. (2008) Emergence of long distance bird migrations: A new model integrating global climate changes. Naturwissenschaften, 95, 1109-1119. doi:10.1007/s00114-008-0435-3 